\title{
Character Association and Path Coefficient Analysis of Grain Yield and Yield Components in Maize (Zea mays L.)
}

\author{
G. Usha Rani ${ }^{*}$, V. Satyanarayana Rao ${ }^{1}$, M. Lal Ahmad ${ }^{1}$ and K.L. Narasimha Rao ${ }^{2}$ \\ ${ }^{1}$ Department of Genetics and Plant Breeding, Agricultural College, Bapatla, A.P., India \\ ${ }^{2}$ Department of Plant Physiology, Agricultural College, Bapatla, A.P., India \\ *Corresponding author
}

\section{A B S T R A C T}

\begin{tabular}{|l|}
\hline Ke y w or d s \\
Character \\
association, \\
Path analysis, \\
Yield, Maize.
\end{tabular}

Correlation studies with 21 maize hybrids indicated that cob length, number of kernel rows per cob, 100 seed weight, and Harvest index showed significant positive association with grain yield per plant both at phenotypic and genotypic levels. Path analysis studies revealed that biological yield and harvest index exerted maximum positive direct effect followed by cob length, plant height and protein content on grain yield per plant and grain yield per ha.

\section{Introduction}

Grain yield, being the most important and complex character governed by quantitative genes and is much more influenced by environmental factors in which the plant is grown. Therefore selection based on only yield performance may create confusion and give a biased result. A study on the nature and degree of association of yield contributing component traits with yield assumes greater importance for fixing up characters that are likely to play a decisive role in influencing yield. The knowledge of interrelationship between yield and its components themselves are useful, if selection for simultaneous improvement in these characters is to be effective. As more variables are included in the correlation study, the associations become more complex. In such a situation, the path coefficient analysis provides an effective means of finding out direct and indirect causes and effects of association and permits a critical examination of the specific forces acting to produce a given correlation and measures the relative importance of each factor. Therefore, the present study on maize was conducted to study the correlation and path coefficients.

\section{Materials and Methods}

The experimental material for the present study consisted of twenty one maize hybrids obtained from Directorate of Maize Research. The hybrids were evaluated in a randomized 
block design with three replications in three environments viz., kharif, 2011, late kharif 2011 and rabi 2011- 12 at Agricultural College Farm, Bapatla, Andhra Pradesh. Each entry was sown in three rows of 5 meter length spaced at $75 \mathrm{~cm}$ and a plant to plant spacing of $25 \mathrm{~cm}$ was maintained. Data was recorded on ten randomly selected plants for each hybrid in each location. The data generated over seasons was pooled and analyzed for estimating the correlation coefficients suggested by Snedecor and Cochran, (1965) and direct and indirect effects of yield components on yield as suggested by Dewey and Lu (1959).

\section{Results and Discussion}

The phenotypic and genotypic correlations among the yield and yield component characters in maize were presented table 1. Correlation studies revealed that genotypic correlation coefficients were higher than phenotypic correlation coefficients for most of the characters under study indicating the strong inherent association between the characters which governed largely by genetic causes and generally less subjected to environmental forces.

Genotypic correlation revealed the existence of real association whereas the phenotypic correlations may occur by chance. The low phenotypic correlations could have resulted due to the modifying effect of environment on the association of characters at the genotypic level.

Grain yield per plant was found to be significantly and positively associated with cob length $\left(0.7866^{* *} ; 0.3864 * *\right)$, number of kernel rows per cob $\left(0.4066^{* * ;} 0.4272^{* *}\right)$, 100 grain weight $(0.7692 * * ; 0.4160 * *)$ and harvest index $(0.4182 * * ; 0.4160 * *)$ both at phenotypic and genotypic levels while protein content $\left(0.2498^{*}\right)$ only at genotypic level. Plant height had significant positive association with 100 seed weight, number of kernel rows per cob but it negative association with grain yield per plant. Number of kernel rows per cob and 100 grain weight showed significant positive association with cob length, harvest index, protein content both at phenotypic and genotypic levels. However, characters which were correlated genotypically but not phenotypically may not be of practical value in selection since selection is based on phenotypes as observed in case of relationship between biological yield and grain yield. Similar findings were earlier noted for cob length, number of kernel rows per cob, 100 seed weight, Ei-Shouny et al., (2005), Shelake et al., (2005), Sofi and Rather (2007), Brar et al., (2008), Hemavathy et al., (2008), Lone e al., (2010), Wannows et al., (2010), Golam et al., (2011) and Jawaharlal et al., (2011).

Estimates of direct and indirect effects of individual characters towards grain yield are presented in table 2 and 3. The path coefficients revealed largest direct effects of biological yield and harvest index on yield followed by cob length and protein content on grain yield. The high direct effect of these traits appeared to be the main factor their strong association with grain yield. Hence, direct selection for these traits would be effective. Plant height exhibited positive direct effect and its indirect effect through days to $50 \%$ silking and biological yield per plant. Cob length influenced yield by positive direct effect and its influence through other characters like biological yield and harvest index. Number of kernel rows per cob showed positive direct effect and influence through 100 seed weight, biological yield and harvest index on Grain yield per plant at phenotypic level while in genotypic level it had negative direct effect. Protein content showed positive direct effect on grain yield per plant and indirect effects through biological yield and lysine content. 


\section{Int.J.Curr.Microbiol.App.Sci (2017) 6(12): 4044-4050}

Table.1 Phenotypic and genotypic correlation among 15 characters in maize (Zea mays L.) in pooled environment

\begin{tabular}{|c|c|c|c|c|c|c|c|c|c|c|c|c|c|c|}
\hline Character & $\begin{array}{r}\text { Plant } \\
\text { height } \\
(\mathrm{cm})\end{array}$ & $\begin{array}{r}\text { Days to } \\
50 \% \\
\text { tasseling }\end{array}$ & $\begin{array}{r}\text { Days to } \\
50 \% \\
\text { silking }\end{array}$ & $\begin{array}{r}\text { Cob } \\
\text { length } \\
\text { (cm) }\end{array}$ & $\begin{array}{r}\text { Kernel } \\
\text { rows/ } \\
\text { cob }\end{array}$ & $\begin{array}{r}100 \text { seed } \\
\text { weight }(\mathrm{g})\end{array}$ & $\begin{array}{r}\text { Biological } \\
\text { yield } \\
\text { (g) }\end{array}$ & $\begin{array}{r}\text { Harvest } \\
\text { index } \\
(\%)\end{array}$ & $\begin{array}{r}\text { Stay green } \\
\text { nature }\end{array}$ & $\begin{array}{l}\text { Protein } \\
\text { content } \\
(\%)\end{array}$ & $\begin{array}{r}\text { Tryptopha } \\
\text { n content } \\
(\%)\end{array}$ & $\begin{array}{r}\text { Lysine } \\
\text { content } \\
(\%)\end{array}$ & $\begin{array}{r}\text { Oil content } \\
(\%)\end{array}$ & $\begin{array}{l}\text { Grain } \\
\text { yield/ plant } \\
\text { (g) }\end{array}$ \\
\hline Plant height $(\mathrm{cm})$ & 1.0000 & 0.0966 & 0.1341 & -0.1103 & 0.0078 & $0.1888^{* *}$ & $0.1921 * *$ & $-0.2098 * *$ & -0.0603 & -0.0376 & 0.1076 & 0.1235 & 0.0960 & -0.0192 \\
\hline Days to $50 \%$ tasseling & 0.1543 & 1.0000 & $0.9064 * *$ & 0.0081 & -0.0162 & 0.0352 & -0.0628 & 0.0145 & 0.0138 & $-0.1926 * *$ & 0.0695 & 0.0631 & -0.0471 & -0.0068 \\
\hline Days to $50 \%$ silking & 0.1533 & $0.9840 * *$ & 1.0000 & -0.0794 & 0.0103 & 0.0892 & -0.0288 & 0.0065 & 0.0125 & $-0.1438^{*}$ & 0.0686 & 0.0542 & 0.0168 & 0.0150 \\
\hline Cob length $(\mathrm{cm})$ & $-0.2729 *$ & -0.0014 & -0.0428 & 1.0000 & $0.3413^{* *}$ & $0.1526^{*}$ & $0.2445 * *$ & $0.1466^{*}$ & -0.0020 & -0.0668 & 0.0892 & 0.0655 & -0.0319 & $0.3864 * *$ \\
\hline Kernel rows/ cob & 0.0005 & 0.1033 & 0.0814 & $0.6598^{* * *}$ & 1.0000 & $0.3534 * *$ & 0.1305 & $0.2501^{* *}$ & $0.1508^{*}$ & 0.1398 & 0.0634 & 0.0839 & 0.0265 & $0.4272 * *$ \\
\hline 100 seed weight $(\mathrm{g})$ & $0.4383^{* *}$ & 0.0473 & 0.1026 & 0.1469 & $0.9264 * *$ & 1.0000 & $0.2209^{* *}$ & $0.1495^{*}$ & 0.0507 & 0.1369 & $0.1809^{*}$ & $0.1954^{* *}$ & $0.3017 * *$ & $0.4160^{* *}$ \\
\hline Biological yield (g) & $0.3880 * *$ & -0.0671 & -0.0327 & 0.0955 & 0.0550 & 0.1502 & 1.0000 & $-0.4050 * *$ & -0.0845 & 0.0969 & 0.0032 & 0.0014 & 0.0261 & $0.5966^{*}$ \\
\hline Harvest index (\%) & $\begin{array}{c}- \\
0.3483^{* *}\end{array}$ & 0.0185 & -0.0088 & $0.4179^{* *}$ & $0.8598^{* *}$ & $0.3972 * *$ & $-0.7818 * *$ & 1.0000 & $0.1613^{*}$ & -0.0013 & 0.0145 & 0.0357 & 0.0902 & $0.4109 * *$ \\
\hline Staygreen nature & -0.1654 & 0.0406 & 0.0170 & 0.1731 & $0.4408^{* *}$ & 0.1359 & $-0.2824 * *$ & $0.3373^{* *}$ & 1.0000 & $0.2912 * *$ & $0.3095^{* *}$ & $0.3247^{* *}$ & $0.2231 * *$ & 0.0492 \\
\hline Protein content $(\%)$ & -0.0744 & -0.2196 & -0.1693 & -0.0257 & $0.3706^{* *}$ & $0.3235^{* *}$ & 0.1808 & -0.0086 & $0.4739 * *$ & 1.0000 & $0.6488 * *$ & $0.6779 * *$ & $0.4335 * *$ & 0.0850 \\
\hline Tryptophan content $(\%)$ & 0.1409 & 0.0655 & 0.0692 & 0.1571 & 0.1609 & $0.2463^{*}$ & -0.0066 & 0.0171 & $0.5234 * *$ & $0.7322 * *$ & 1.0000 & $0.9682 * *$ & $0.5386 * *$ & 0.0272 \\
\hline Lysine content (\%) & 0.1554 & 0.0651 & 0.0642 & 0.1871 & 0.2100 & $0.2915^{*}$ & 0.0224 & 0.0193 & $0.5256^{* *}$ & $0.7407 * *$ & $0.9965^{* *}$ & 1.0000 & $0.5448 * *$ & 0.0419 \\
\hline Oil content (\%) & 0.1213 & -0.0614 & 0.0266 & -0.1258 & $0.2408^{*}$ & $0.5239^{* *}$ & 0.0217 & 0.1066 & $0.4264 * *$ & $0.5420^{* *}$ & $0.6220^{* *}$ & $0.6348 * *$ & 1.0000 & 0.0935 \\
\hline Grain yield/ plant (g) & -0.0204 & 0.0300 & 0.0489 & $0.7686 * *$ & $0.4066 * *$ & $0.7692 * *$ & 0.1917 & $0.4182 * *$ & 0.1943 & $0.2498 *$ & 0.0496 & 0.0913 & 0.2161 & 1.0000 \\
\hline
\end{tabular}

* Significant at 0.05 level, ** Significant at 0.01 level 


\section{Int.J.Curr.Microbiol.App.Sci (2017) 6(12): 4044-4050}

Table.2 Direct and indirect (genotypic) effects of 15 characters on seed yield per plant in pooled environment

\begin{tabular}{|c|c|c|c|c|c|c|c|c|c|c|c|c|c|}
\hline Character & $\begin{array}{c}\text { Plant } \\
\text { height } \\
(\mathrm{cm})\end{array}$ & $\begin{array}{c}\text { Days to } \\
\mathbf{5 0 \%} \\
\text { tasseling }\end{array}$ & $\begin{array}{c}\text { Days to } \\
\mathbf{5 0 \%} \\
\text { silking }\end{array}$ & $\begin{array}{c}\text { Cob } \\
\text { length } \\
(\mathrm{cm})\end{array}$ & $\begin{array}{c}\text { Kernel } \\
\text { rows/ } \\
\text { cob }\end{array}$ & $\begin{array}{c}100 \text { seed } \\
\text { weight } \\
\text { (g) }\end{array}$ & $\begin{array}{l}\text { Biological } \\
\text { yield } \\
\text { (g) }\end{array}$ & $\begin{array}{c}\text { Harvest } \\
\text { index } \\
(\%)\end{array}$ & $\begin{array}{c}\text { Stay green } \\
\text { nature }\end{array}$ & $\begin{array}{c}\text { Protein } \\
\text { content } \\
(\%)\end{array}$ & $\begin{array}{c}\text { Tryptoph } \\
\text { an } \\
\text { content } \\
(\%)\end{array}$ & $\begin{array}{c}\text { Lysine } \\
\text { content } \\
(\%)\end{array}$ & $\begin{array}{c}\text { Oil } \\
\text { content } \\
(\%)\end{array}$ \\
\hline Plant height $(\mathrm{cm})$ & 0.2242 & 0.0346 & 0.0344 & -0.0612 & 0.0001 & 0.0983 & 0.0870 & -0.0781 & -0.0371 & -0.0167 & 0.0316 & 0.0348 & 0.0272 \\
\hline Days to $50 \%$ tasseling & -0.0373 & -0.2417 & -0.2378 & 0.0003 & -0.0250 & -0.0114 & 0.0162 & -0.0045 & -0.0098 & 0.0531 & -0.0158 & -0.0157 & 0.0148 \\
\hline Days to $50 \%$ silking & 0.0656 & 0.4211 & 0.4280 & -0.0183 & 0.0349 & 0.0439 & -0.0140 & -0.0038 & 0.0073 & -0.0724 & 0.0296 & 0.0275 & 0.0114 \\
\hline Cob length (cm) & -0.0743 & -0.0004 & -0.0117 & 0.2722 & 0.1796 & 0.0400 & 0.0260 & 0.1138 & 0.0471 & -0.0070 & 0.0428 & 0.0509 & -0.0343 \\
\hline Kernel rows/ cob & -0.0001 & -0.0131 & -0.0104 & -0.0840 & -0.1273 & -0.1179 & -0.0070 & -0.1094 & -0.0561 & -0.0472 & -0.0205 & -0.0267 & -0.0306 \\
\hline 100 seed weight $(\mathrm{g})$ & -0.0842 & -0.0091 & -0.0197 & -0.0282 & -0.1781 & -0.1922 & -0.0289 & -0.0763 & -0.0261 & -0.0622 & -0.0473 & -0.0560 & -0.1007 \\
\hline Biological yield (g) & 0.4836 & -0.0836 & -0.0408 & 0.1190 & 0.0685 & 0.1872 & 1.2464 & -0.9745 & -0.3519 & 0.2253 & -0.0082 & 0.0279 & 0.0271 \\
\hline Harvest index $(\%)$ & -0.5287 & 0.0280 & -0.0134 & 0.6343 & 1.3051 & 0.6029 & -1.1868 & 1.5179 & 0.5119 & -0.0131 & 0.0260 & 0.0293 & 0.1618 \\
\hline Stay green nature & -0.0128 & 0.0031 & 0.0013 & 0.0134 & 0.0340 & 0.0105 & -0.0218 & 0.0260 & 0.0771 & 0.0366 & 0.0404 & 0.0405 & 0.0329 \\
\hline Protein content $(\%)$ & -0.0255 & -0.0753 & -0.0581 & -0.0088 & 0.1271 & 0.1110 & 0.0620 & -0.0030 & 0.1625 & 0.3430 & 0.2511 & 0.2541 & 0.1859 \\
\hline Tryptophan content (\%) & -0.0855 & -0.0397 & -0.0420 & -0.0953 & -0.0976 & -0.1494 & 0.0040 & -0.0104 & -0.3175 & -0.4441 & -0.6066 & -0.6045 & -0.3773 \\
\hline Lysine content (\%) & 0.0365 & 0.0153 & 0.0151 & 0.0439 & 0.0493 & 0.0684 & 0.0052 & 0.0045 & 0.1233 & 0.1738 & 0.2338 & 0.2347 & 0.1490 \\
\hline Oil content $(\%)$ & 0.0181 & -0.0091 & 0.0040 & -0.0187 & 0.0359 & 0.0780 & 0.0032 & 0.0159 & 0.0635 & 0.0807 & 0.0926 & 0.0945 & 0.1489 \\
\hline Grain yield/ plant (g) & -0.0204 & 0.0300 & 0.0489 & 0.7686 & 0.4066 & 0.7692 & 0.1917 & 0.4182 & 0.1943 & 0.2498 & 0.0496 & 0.0913 & 0.2161 \\
\hline Grain yield/ ha (q) & -0.0046 & -0.0073 & 0.0209 & 0.2092 & -0.1790 & -0.1479 & 0.2389 & 0.6348 & 0.0150 & 0.0857 & -0.0301 & 0.0214 & 0.0322 \\
\hline
\end{tabular}

Note: Bold figures indicates direct effects, Residual effect $=0.3325$

** Significant at $1 \%$ probability level

* Significant at $5 \%$ probability level 
Int.J.Curr.Microbiol.App.Sci (2017) 6(12): 4044-4050

Table.3 Direct and indirect (phenotypic) effects of 15 characters on seed yield per plant in pooled environment

\begin{tabular}{|c|c|c|c|c|c|c|c|c|c|c|c|c|c|}
\hline Character & $\begin{array}{c}\text { Plant } \\
\text { height } \\
(\mathrm{cm})\end{array}$ & $\begin{array}{c}\text { Days to } \\
\mathbf{5 0 \%} \\
\text { tasseling }\end{array}$ & $\begin{array}{c}\text { Days to } \\
\mathbf{5 0 \%} \\
\text { silking }\end{array}$ & $\begin{array}{c}\text { Cob } \\
\text { length } \\
(\mathrm{cm})\end{array}$ & $\begin{array}{c}\text { Kernel } \\
\text { rows/ } \\
\text { cob }\end{array}$ & $\begin{array}{c}100 \text { seed } \\
\text { weight (g) }\end{array}$ & $\begin{array}{c}\text { Biologica } \\
\text { l yield } \\
\text { (g) }\end{array}$ & $\begin{array}{c}\text { Harvest } \\
\text { index } \\
(\%)\end{array}$ & $\begin{array}{c}\text { Stay green } \\
\text { nature }\end{array}$ & $\begin{array}{c}\text { Protein } \\
\text { content } \\
(\%)\end{array}$ & $\begin{array}{c}\text { Tryptoph } \\
\text { an } \\
\text { content } \\
(\%)\end{array}$ & $\begin{array}{c}\text { Lysine } \\
\text { content } \\
(\%)\end{array}$ & $\begin{array}{c}\text { Oil } \\
\text { content } \\
(\%)\end{array}$ \\
\hline Plant height $(\mathrm{cm})$ & -0.0599 & -0.0058 & -0.0080 & 0.0066 & -0.0005 & -0.0113 & -0.0115 & 0.0126 & 0.0036 & 0.0023 & -0.0064 & -0.0074 & -0.0057 \\
\hline Days to $50 \%$ tasseling & 0.0039 & 0.0401 & 0.0363 & 0.0003 & -0.0006 & 0.0014 & -0.0025 & 0.0006 & 0.0006 & -0.0077 & 0.0028 & 0.0025 & -0.0019 \\
\hline Days to $50 \%$ silking & -0.0008 & -0.0056 & -0.0061 & 0.0005 & -0.0001 & -0.0005 & 0.0002 & 0.0000 & -0.0001 & 0.0009 & -0.0004 & -0.0003 & -0.0001 \\
\hline Cob length $(\mathrm{cm})$ & -0.0011 & 0.0001 & -0.0008 & 0.0104 & 0.0036 & 0.0016 & 0.0025 & 0.0015 & 0.0000 & -0.0007 & 0.0009 & 0.0007 & -0.0003 \\
\hline Kernel rows/ cob & 0.0008 & -0.0017 & 0.0011 & 0.0362 & 0.1060 & 0.0375 & 0.0138 & 0.0265 & 0.0160 & 0.0148 & 0.0067 & 0.0089 & 0.0028 \\
\hline 100 seed weight $(\mathrm{g})$ & 0.0181 & 0.0034 & 0.0086 & 0.0146 & 0.0339 & 0.0959 & 0.0212 & 0.0143 & 0.0049 & 0.0131 & 0.0173 & 0.0187 & 0.0289 \\
\hline Biological yield $(\mathrm{g})$ & 0.1653 & -0.0540 & -0.0248 & 0.2104 & 0.1123 & 0.1901 & 0.8608 & -0.3486 & -0.0727 & 0.0834 & 0.0028 & 0.0012 & 0.0225 \\
\hline Harvest index (\%) & -0.1483 & 0.0103 & 0.0046 & 0.1036 & 0.1768 & 0.1057 & -0.2863 & 0.7069 & 0.1140 & -0.0009 & 0.0103 & 0.0252 & 0.0638 \\
\hline Stay green nature & 0.0008 & -0.0002 & -0.0002 & 0.0000 & -0.0020 & -0.0007 & 0.0011 & -0.0022 & -0.0136 & -0.0040 & -0.0042 & -0.0044 & -0.0030 \\
\hline Protein content $(\%)$ & 0.0009 & 0.0046 & 0.0035 & 0.0016 & -0.0034 & -0.0033 & -0.0023 & 0.0000 & -0.0070 & -0.0241 & -0.0157 & -0.0164 & -0.0105 \\
\hline Tryptophan content (\%) & 0.0008 & 0.0005 & 0.0005 & 0.0007 & 0.0005 & 0.0013 & 0.0000 & 0.0001 & 0.0023 & 0.0048 & 0.0073 & 0.0071 & 0.0039 \\
\hline Lysine content (\%) & 0.0017 & 0.0009 & 0.0008 & 0.0009 & 0.0012 & 0.0027 & 0.0000 & 0.0005 & 0.0046 & 0.0095 & 0.0136 & 0.0140 & 0.0076 \\
\hline Oil content $(\%)$ & -0.0014 & 0.0007 & -0.0002 & 0.0005 & -0.0004 & -0.0044 & -0.0004 & -0.0013 & -0.0033 & -0.0063 & -0.0078 & -0.0079 & -0.0146 \\
\hline Grain yield/ plant (g) & -0.0192 & -0.0068 & 0.0150 & 0.3864 & 0.4272 & 0.4160 & 0.5966 & 0.4109 & 0.0492 & 0.0850 & 0.0272 & 0.0419 & 0.0935 \\
\hline Grain yield/ ha (q) & 0.0012 & -0.0003 & -0.0001 & 0.0040 & 0.0453 & 0.0399 & 0.5135 & 0.2905 & -0.0007 & -0.0021 & 0.0002 & 0.0006 & -0.0014 \\
\hline
\end{tabular}

Note: Bold figures indicates direct effects, Residual effect $=0.3305$

** Significant at $1 \%$ probability level ${ }^{`}$

* Significant at 5\% probability level 
Further, number of kernel rows per cob, 100 seed weight and tryptophan content recorded negative direct effects on grain yield. The correlation of cob length, number of kernel rows per cob and 100 seed weight with grain yield was positive indicating the ineffectiveness of direct selection for the trait. Results were in accordance with Chandramohan (1999), Nagesh Kumar and Sudheer Kumar (2000). These for plant height the direct effects was positive, while its association with grain yield was observed to be negative, indicating the importance of restricted selection model (Singh and Kakar, 1977), for exploitation of the direct effects noticed.

A perusal of the results obtained from character association and path coefficient analysis, revealed that cob length, number of number of kernel rows per cob, 100 seed weight and harvest index were found to have significant influence on grain yield, and also have high positive direct and indirect effects through many other characters. Hence, simultaneous selection based on cob length, number of kernel rows per cob, 100 seed weight and harvest index seems to be more promising in improving the grain yield in maize.

\section{References}

Brar, S.P.S., Chawla, J.S and Singh, P. 2008. Studies on different selection indices and path analysis in maize. Crop Improvement. 31(2): 16-19.

Chandra Mohan, Y., 1999. Variability studies for oil and grain yield in maize genotypes. M.Sc. (Ag) thesis submitted to ANGRAU. Agricultural University, Rajendra Nagar, Hyderabad-30.

Dewey, D., and Lu, K.H. 1959. A correlation and path coefficient analysis of components of crested wheat grass seed production. Agronomy Journal. 51:515-
518.

Ei-Shouny, K.A., Ei-Bagoury, O.H., Ibrahim, K.I.M and Al-Ahmed, S.A. 2005. Correlation and path coefficient analysis in four yellow maize crosses under two planting dates. Arab University Journal of Agricultural Sciences. 13(2): 327339.

Golam, F., Farhana, an., Mohammad, F.Z., Nazia, A.M., Rahman, M.M., Rahman, M and Mihdzar, A.K. 2011. Grain yield and associated traits of maize (Zea mays. L.) genotypes in Malaysian tropical environment. African Journal of Agricultural Research. 6(28): 61476154.

Hemavathy, A.T., Balaji, k., Ibrahim, S.M., Anand, G and Sankar, D. 2008. Genetic variability and correlation studies in maize (Zea mays L.). Agricultural Science Digest. 28 (2): 112-114.

Jawaharlal, J., Reddy, G.L and Kumar, R.S. 2011. Genetic variability and character association studies in maize. Agricultural Science Digest. 31(3): 173177

Lone, A.A., Warsi, M.Z.K., Nehvi, F.A and Dar, S.A. 2010. Studies on character association in winter maizeunder normal and excess soil moisture (ESM). Maize Genatic Corporation Newsletter. 84.

Nagesh Kumar, M. V., and Sudheer kumar, S. 2000. Studies on character association and path coefficients for grain yield and oil content in maize. Annals of Agricultural Research. 21(1): 73-78.

Shelake, D.V., Bhave, S.G., Bendale, V.W., Madav, R.R and Pethe, U. B. 2005. Genetic factors influencing grain yield in maize. Journal of Ecobiology. 17(6): 521-528.

Snedecor, G.W., and Cochran, W.G. 1967. Statistical Methods. The Iowa State College Press, Ames, Iowa. U.S.A. 160413. 
Sofi, P.A., and Rather, A.G. 2007. Studies on genetic variability, correlation and path analysis in maize (Zea mays L.). Maize Genetics Co-operation Newsletters.81: 26-27.

Wannous, A.A., Azzam, H.K and Ahmad
S.A. 2012. Genetic variances, heritability, correlation and path coefficient analysis in yellow maize crosses (Zea mays L.). Agriculture and Biology Journal of North America. 1(4): 630-637.

\section{How to cite this article:}

Usha Rani, G., V. Satyanarayana Rao, M. Lal Ahmad and Narasimha Rao, K.L. 2017. Character Association and Path Coefficient Analysis of Grain Yield and Yield Components in Maize (Zea mays L.). Int.J.Curr.Microbiol.App.Sci. 6(12): 4044-4050. doi: https://doi.org/10.20546/ijcmas.2017.612.465 